\title{
Natural orifice transluminal endoscopic surgery (NOTES): implications for anesthesia Michael Schaefer
}

\author{
Address: Department of Anesthesiology and Intensive Care Medicine, Charité University Berlin, Campus Virchow Klinikum, \\ Augustenburgerplatz 1, 13353 Berlin, Germany \\ Email: micha.schaefer@charite.de
}

Fl000 Medicine Reports 2009, I:80 (doi:I0.34I0/MI-80)

The electronic version of this article is the complete one and can be found at: http://FI000.com/Reports/Medicine/content/I/80

\begin{abstract}
Natural orifice transluminal endoscopic surgery (NOTES) has recently evolved as a novel approach for abdominal surgery with great potential to further improve the advantages of laparoscopy over laparotomy. The first patients undergoing NOTES cholecystectomy or appendectomy reported no or only minimal pain, required no narcotic analgesics, and were discharged early from the hospital and immediately resumed daily activities. If this is confirmed by randomized controlled clinical trials, what are the potential implications for anesthesia?
\end{abstract}

\section{Introduction and context}

A unique approach to accessing the abdominal cavity for surgical interventions has emerged over the last few years. Historically, abdominal access has been obtained first via laparotomy and later via laparoscopy. The unique approach of natural orifice transluminal endoscopic surgery (NOTES) does not accept the limitation of the luminal wall as a barrier; instead, it chooses a transgastric (preferred in the US) or a transvaginal (preferred in Europe) passage [1]. In the ideal NOTES situation, all necessary instruments are maneuvered solely through this one access site; however, at present, NOTES is performed as a hybrid approach (that is, an endoscopic transluminal surgery under laparoscopic supervision and/or assistance). Interdisciplinary teams founded by surgeons, gastroenterologists, and the industry - for example, in the US, the Natural Orifice Surgery Consortium for Assessment and Research, and in Europe, the EURO-NOTES Foundation (established in cooperation between the European Association for Endoscopic Surgery and the European Society of Gastrointestinal Endoscopy) - have initiated strategies to cope with the fundamental challenges of this new approach, which they hope will replace laparoscopic surgery sometime in the future. Typical of such challenges are improved technical equipment, easy manipulation of target organs, safe closure of the viscerotomy, and prevention of infections at the natural orifice [2]. Although currently this new surgical approach only concerns anesthesiologists at specialized centers, experience with laparoscopic surgery tells us that anesthesiologists may be using this technique sooner rather than later.

What are the potential benefits of NOTES? Patients undergoing a NOTES procedure (e.g., removal of the gallbladder) will have, instead of an abdominal wall incision, a relatively small perforation of the gastric or vaginal wall which can be closed by only a few stitches [1]. The lack of an abdominal wall incision will avoid prolonged wound healing, visible scaring, and hernia formation. In addition, patients will probably have a reduced need for anesthesia, less postoperative pain, and an earlier recovery time. A lot of experience and improvement of this technique has been gained in animal studies; however, only about 30 cases of NOTES procedures in patients have been done worldwide [2].

Anesthesiologists have important questions that need to be answered: What were the experiences in these few patients with regard to anesthesia and postoperative 
pain? Is there a difference between visceral and somatic pain? Is regional anesthesia likely to be an option for NOTES? If patients had a choice, what procedure would they favor?

\section{Recent advances}

\section{Benefits of laparoscopy over laporotomy}

Since NOTES is an advancement of laparoscopic surgery and since data with regard to NOTES are scarce, the question arises as to whether laparoscopy compared with laparotomy has already shown a reduction in postoperative pain or analgesic consumption. Consistent with common clinical experience, the multicenter COLOR (COlon cancer Laparoscopic or Open Resection) trial showed that the 536 patients receiving laparoscopy for colon cancer surgery consumed significantly less opioid, nonopioid, or epidural analgesics during the first three postoperative days and had a shorter hospital stay compared with the 546 patients undergoing laparotomy [3]. In a 5-year follow-up of another randomized trial to assess pain and numbness after laparoscopic or open repair of groin hernia, significantly fewer patients reported pain and numbness in the groin 12 and 36 months following laparoscopy [4]. Consistent with these and other clinical trials and also with daily clinical practice, the majority of laparoscopic surgeries are still performed under general anesthesia at present.

\section{Benefits of NOTES over laparoscopy}

For several years, endoscopic drainage of pancreatic pseudocysts and transgastric debridement of peripancreatic necroses have been standard procedures for experienced endoscopists [5]. Patients recovered fairly quickly and often showed immediate pain relief. Stimulated by a report in which NOTES was described for the first time in an animal model as the potential next step of endoscopic surgery [6], the first implementations of NOTES procedures in patients slowly evolved. In one of the first published cases, a 66-year-old woman underwent a laparoscopically assisted transvaginal cholecystectomy under general anesthesia lasting 3.5 hours [7]. A laparoscope was placed at the umbilicus and used only intermittently for video documentation and carbon dioxide insufflation. The gall bladder was successfully removed from the abdomen through the vaginal incision. The patient recovered fairly well after surgery without any complications and was discharged the next day. In another case of a 30-year-old woman undergoing cholecystectomy, transvaginal access to the peritoneal cavity was gained under laparoscopic guidance [8]. After 3 hours of surgery in general anesthesia, the gallbladder was removed transvaginally and the colpotomy was closed. The patient recovered immediately after surgery, with no postoperative pain and no scars, and was discharged the next day. At a follow-up visit 10 days later, the patient had resumed daily life activity and had no discomfort at the vaginal access site.

These single reports were further substantiated only recently by a series of 11 successful NOTES procedures that lasted between 70 and 150 minutes: nine transvaginal cholecystectomies, one transgastric appendectomy, and one transvaginal appendectomy were performed [9]. None of the patients, except one who had a transvaginal approach, required narcotic pain medications. All patients were discharged on the first postoperative day. No complications were reported at 1-week or 1-month follow-up visits. A second publication this year reported 10 transvaginal and 6 transgastric cholecystectomies with very similar results: immediate recovery of patients, minimal postoperative pain intensity (visual analog scores of $2 / 10$ at 24 hours and $0 / 10$ at 48 hours) and minimal analgesic requirements [10]. Taken together, the first clinical experiences with patients undergoing NOTES procedures such as cholecystectomies and appendectomies demonstrate that postoperative pain intensity is minimal and does not require narcotic medications typically given after these procedures. As a result, patients were able to leave the hospital the next day. The advantages of laporoscopy seem to be further enhanced by NOTES. However, these findings need to be corroborated by further randomized controlled clinical trials comparing natural orifice surgery with laparoscopic surgery.

\section{Differences between somatic and visceral pain}

The results from the first clinical reports about NOTES suggest differences between visceral and somatic pain. Indeed, somatic pain following skin injury is usually sharp and stabbing, is well localized, and can be evoked from all skin areas. However, visceral pain is dull and nagging and poorly localized, and some viscera (for example, liver and spleen) do not even give rise to pain unless the organ is inflamed [11]. Visceral and skin afferent neurons encode different qualities of sensory information: sensations of organ filling, bloating, and distension of the viscera are in sharp contrast to sensations of touch, pinch, heat, and vibration of the skin.

Recent evidence from experimental studies sees major differences in the innervation of the gut compared with the skin [11]. The ability to identify the source of pain is excellent in the skin, generally good in muscles and deeper tissues, but poor and diffuse in the viscera. In contrast to the dense and somatotopically oriented innervation of the skin, the viscera are only sparsely innervated. Fewer than $7 \%$ of afferent nerves entering the spinal cord project to the viscera, and only a small 
fraction of these transmit sensory input to the central nervous system [11]. Further studies need to show whether the sparse innervation of the viscera is contributing to the lack of severe pain following abdominal NOTES procedures.

In the spinal cord, sensory afferents coming from the viscera arborize widely over several segments, even to the contralateral side, which may explain the wide distribution and diffuse character of visceral pain. Innervation of the vaginal wall, for example, spreads via the pelvic and hypogastric nerves over several spinal segments (L2-S2), and innervation of the gastric wall expands over even more spinal segments (T1-L4). This will preclude the possibility that some enthusiastic proponents of NOTES already envision, namely that entire NOTES procedures can be done by local anesthesia at the access site.

\section{Is regional anesthesia an option?}

Can it be imagined that spinal or epidural anesthesia, blocking sensory input over several spinal segments, is applicable for NOTES procedures? Under certain conditions, the answer is yes; however, there are still several limitations to this anesthetic regimen. Clinical experience shows that laparoscopic procedures, and more so at the moment NOTES procedures, last longer than traditional laparotomy. For example, in the multicenter COLOR trial, the mean duration time for the laporoscopic colon resection was between 30 and 150 minutes longer at most of the 21 participating centers than for open colon resection [3]. This may challenge patients' endurance during the surgical procedure. Moreover, patients are often put in uncomfortable positions such as head-down or Trendelenburg position to facilitate visualization, which should be limited to short periods of time to be tolerable.

Laparoscopy and also NOTES procedures require the establishment of a pneumoperitoneum, which influences several physiologic functions [12]. Carbon dioxide insufflation accumulates in the circulation via absorption through the peritoneum, resulting in elevated arterial partial pressure of carbon dioxide $\left(\mathrm{pCO}_{2}\right)$ and decreased arterial $\mathrm{pH}$, which may be harmful if not compensated by an increase in minute ventilation [12]. These alterations may be accentuated in patients with chronic cardiopulmonary disease [13]. Increasing pressure in the abdominal cavity compromises movement of the diaphragm and diminishes pulmonary function parameters such as FEV1 (forced expiratory volume in 1 second) and FVC (forced vital capacity). This may lead to the formation of alveolar atelectases and resultant ventilation/perfusion mismatch [12]. Finally, hemodynamic parameters are affected via direct and indirect effects on the cardiovascular system. Pneumoperitoneum initially may lead to venous compression, decreased venous blood return, and subsequent diminished cardiac output, particularly in volume-depleted patients. However, these effects are counteracted by sympathetic stimulation causing tachycardia and systemic vasoconstriction. In a normal patient, these alterations in physiologic functions may not be harmful but they may put patients with compromised cardiopulmonary functions at risk [12]. The intraperitoneal pressure currently used in most hospitals is between 10 and $15 \mathrm{~mm} \mathrm{Hg}$. If this could be reduced to a low pressure range of $5-7 \mathrm{~mm} \mathrm{Hg}$, most of the discussed alterations in physiologic functions would be irrelevant. Moreover, the incidence and severity of postoperative shoulder pain would be significantly lower in patients having low-pressure pneumoperitoneum [14]. In fact, in its clinical practice guidelines, the European Association for Endoscopic Surgery has recommended the use of the lowest intra-abdominal pressure, allowing adequate exposure of the operative field, rather than the use of a routine pressure [15].

Regional anesthesia for laparoscopic surgery is feasible as it has been shown in 4,645 patients at an Indian medical hospital [16]. Most patients underwent laparoscopic cholecystectomy with an intra-abdominal pressure of 8-10 $\mathrm{mm} \mathrm{Hg}$, a mean duration of 15-20 minutes, and an average time to discharge of 2.3 days. Thus, under optimal conditions (that is, short duration, time limit for uncomfortable positions, low intraperitoneal pressure, and noninvasive arterial $\mathrm{pCO}_{2}$ monitoring), regional anesthesia may be an option for NOTES procedures in the future. Right now, however, general anesthesia remains current practice.

\section{Patients' preferences}

When patients are informed about the advantages and possible risks of NOTES, would they consider NOTES as a likely option for surgery? Recently published surveys tried to find an honest answer to this question. After having received a written description of minimally invasive surgery and NOTES surgery, 100 women at a university hospital were given a 10-question survey [17]. The majority of women (68\%) would agree to a transvaginal procedure because they understood that it carries a reduced risk of hernia and postoperative pain. However, only $39 \%$ of women were concerned about cosmetic aspects. Women were very much concerned about the risk of infection (83\%), infertility (61\%), and dyspareunia (81\%). In another survey, 100 patients, who were evaluated for abdominal pain, pancreatitis, or suspected cholcystollithiasis, received a questionnaire in regard to a potential choice for a laparoscopic or NOTES cholecystectomy [18]. Patients who were 
younger than 51 years old, female, and who had prior endoscopic experience were more likely to prefer NOTES. For both men and women who had preferred NOTES, the oral route was the first choice. If potential complications were not more than 3\%, acceptance of NOTES would be at least $97 \%$. However, if potential complications were greater than $3 \%$, acceptance of NOTES would not be more than $15 \%$ [18]. Surveys at the SAGES (Society of American Gastrointestinal Endoscopic Surgeons) 2007 meeting concluded that complications, recovery time, and postoperative pain were considered much more important than cosmetic or economic aspects [2].

\section{Implications for clinical practice}

NOTES has the potential to further improve the advantages of laparoscopy over laparotomy. This becomes most obvious in the first reports of patients undergoing a NOTES procedure who recovered promptly after surgery, had no or only minimal pain, required no narcotic medications, and were discharged the day after surgery. Future randomized controlled clinical trials have to corroborate these results and have to identify patients, such as severely obese patients perhaps, who would greatly benefit from it.

The enthusiasm about these promising results makes some doctors believe that, in the future, NOTES procedures can be done only by local anesthesia at the access site. This is, however, unrealistic since the sensory innervation of the viscera - though sparse - spreads over many spinal segments. A spinal or epidural anesthesia would be more realistic; however, it lies far in the future since several factors of the NOTES procedure still need to be optimized.

For the anesthesiologist, the most important considerations are a short duration of surgery, a minimum time in an uncomfortable position, a reduced intraperitoneal pressure (ideally 5-8 $\mathrm{mm} \mathrm{Hg}$ ), and noninvasive arterial $\mathrm{pCO}_{2}$ monitoring. Together with a low risk of complications, this will finally make NOTES more favorable for patients.

\section{Abbreviations \\ COLOR, COlon cancer Laparoscopic or Open Resection; FEV1, forced expiratory volume in 1 second; FVC, forced vital capacity; NOTES, natural orifice transluminal endoscopic surgery; $\mathrm{pCO}_{2}$, partial pressure of carbon dioxide; SAGES, Society of American Gastrointestinal Endoscopic Surgeons.}

\section{Competing interests}

The author declares that he has no competing interests.

\section{References}

I. McGee MF, Rosen MJ, Marks J, Onders RP, Chak A, Faulx A, Chen VK, Ponsky J: A primer on natural orifice transluminal endoscopic surgery: building a new paradigm. Surg Innov 2006, 13:86-93.

2. Flora ED, Wilson TG, Martin IJ, O'Rourke NA, Maddern GJ: A review of natural orifice translumenal endoscopic surgery (NOTES) for intra-abdominal surgery: experimental models, techniques, and applicability to the clinical setting. Ann Surg 2008, 247:583-602.

3. Veldkamp R, Kuhry E, Hop WC, Jeekel J, Kazemier G, Bonjer HJ, Haglind E, Påhlman L, Cuesta MA, Msika S, Morino M, Lacy AM; COlon cancer Laparoscopic or Open Resection Study Group (COLOR): Laparoscopic surgery versus open surgery for colon cancer: short-term outcomes of a randomized trial. Lancet Oncol 2005, 6:477-84.

4. Grant AM, Scott NW, O'Dwyer PJ; MRC Laparoscopic Groin Hernia Trial Group: Five-year follow-up of a randomized trial to assess pain and numbness after laparoscopic or open repair of groin hernia. Br J Surg 2004, 91 : 1570-4.

5. Seifert H, Wehrmann T, Schmitt T, Zeuzem S, Caspary WF: Retroperitoneal endoscopic debridement for infected peripancreatic necrosis. Lancet 2000, 356:653-5.

6. Kalloo AN, Singh VK, Jagannath SB, Niiyama H, Hill SL, Vaughn CA Magee CA, Kantsevoy SV: Flexible transgastric peritoneoscopy: a novel approach to diagnostic and therapeutic interventions in the peritoneal cavity. Gastrointest Endosc 2004, 60: I I4-7.

7. Bessler M, Stevens PD, Milone L, Parikh M, Fowler D: Transvaginal laparoscopically assisted endoscopic cholecystectomy: a hybrid approach to natural orifice surgery. Gastrointest Endosc 2007, 66:1243-5.

8. Marescaux J, Dallemagne B, Perretta S, Wattiez A, Mutter D, Coumaros D: Surgery without scars: report of transluminal cholecystectomy in a human being. Arch Surg 2007, 142:823-6.

9. Horgan S, Cullen JP, Talamini MA, Mintz Y, Ferreres A, Jacobsen GR, Sandler B, Bosia J, Savides T, Easter DW, Savu MK, Ramamoorthy SL, Whitcomb E, Agarwal S, Lukacz E, Dominguez G, Ferraina P: Natural orifice surgery: initial clinical experience. Surg Endosc 2009, 23:15I2-8.

10. Asakuma M, Perretta S, Allemann P, Cahill R, Con SA, Solano C, Pasupathy S, Mutter D, Dallemagne B, Marescaux J: Challenges and lessons learned from NOTES cholecystectomy initial experience: a stepwise approach from the laboratory to the clinical application. J Hepatobiliary Pancreat Surg 2009, 16:249-54.

II. Robinson DR, Gebhart GF: Inside information: the unique features of visceral sensation. Mol Interv 2008, 8:242-53.

12. Grabowski JE, Talamini MA: Physiological effects of pneumoperitoneum. J Gastrointest Surg 2009, 13:1009-16.

13. Galizia G, Prizio G, Lieto E, Castellano P, Pelosio L, Imperatore V, Ferrara A, Pignatelli C: Hemodynamic and pulmonary changes during open, carbon dioxide pneumoperitoneum and abdominal wall-lifting cholecystectomy. A prospective, randomized study. Surg Endosc 200I, 15:477-83.

14. Gurusamy KS, Samraj K, Davidson BR: Low pressure versus standard pressure pneumoperitoneum in laparoscopic cholecystectomy. Cochrane Database Syst Rev 2009, 2:CD006930.

15. Neudecker J, Sauerland S, Neugebauer E, Bergamaschi R, Bonjer HJ, Cuschieri A, Fuchs KH, Jacobi Ch, Jansen FW, Koivusalo AM, Lacy A, McMahon MJ, Millat B, Schwenk W: The European Association for Endoscopic Surgery clinical practice guideline on the pneumoperitoneum for laparoscopic surgery. Surg Endosc 2002, 16: II2I-43

16. Sinha R, Gurwara AK, Gupta SC: Laparoscopic surgery using spinal anesthesia. JSLS 2008, I2:133-8.

17. Peterson CY, Ramamoorthy S, Andrews B, Horgan S, Talamini M, Chock A: Women's positive perception of transvaginal NOTES surgery. Surg Endosc 2009, 23:1770-4.

18. Varadarajulu S, Tamhane A, Drelichman ER: Patient perception of natural orifice transluminal endoscopic surgery as a technique for cholecystectomy. Gastrointest Endosc 2008, 67:854-60. 